\title{
Correction to: Long-term effect of high-intensity laser therapy in the treatment of patients with chronic low back pain: a randomized blinded placebo-controlled trial
}

\author{
Mohamed Salaheldien Mohamed Alayat ${ }^{1}$ - Azza Mohamed Atya ${ }^{1} \cdot$ Mohamed Mohamed Ebrahim Ali $^{2,3}$. \\ Tamer Mohamed Shousha ${ }^{3}$ \\ Published online: 2 December 2019 \\ C) Springer-Verlag London Ltd., part of Springer Nature 2019
}

\section{Correction to: Lasers in Medical Science.}

https://doi.org/10.1007/s10103-013-1472-5

After publication of this paper, the authors determined that the name of the author Tamer Mohamed Shosha was incorrectly spelled. The correct presentation should be Tamer Mohamed Shousha.

The online version of the original article can be found at https://oi.org/ 10.1007/s10103-013-1472-5

Mohamed Salaheldien Mohamed Alayat

mohsalahpt@hotmail.com

1 Basic Science Department, Faculty of Physical Therapy, Cairo University, 7 Ahmed Elziat Street from Eltahrir Street, Cairo, Egypt

2 Department of Physical Therapy for Musculoskeletal Disorders, Faculty of Physical Therapy, Cairo University, Cairo, Egypt

3 Department of Physical Therapy, Faculty of Applied Medical Sciences, Umm Al-Qura University, Mecca, Saudi Arabia 